\title{
Vascular Changes Following Mucoperiosteal Flap Surgery: A Fluorescein Angiography Study in Dogs
}

Thomas N. McLean ${ }^{*}$ Billy A. Smith, ${ }^{\dagger}$ Edith C. Morrison, ${ }^{\ddagger}$ Carlos E. Nasjleti, ${ }^{\ddagger}$ and

Raul G. Caffesse

THIS STUDY WAS UNDERTAKEN TO DETERMINE the vascular changes which occur following mucoperiosteal flap surgery where two different suturing techniques were employed. In four healthy adult mongrel dogs, buccal and lingual full-thickness envelope flaps were reflected in the mandibular quadrants following intracrevicular incisions from the first premolar to the first molar. The flaps were immediately readapted and primary closure was achieved by the horizontal mattress suturing technique in one quadrant and the direct interrupted suturing technique in the contralateral quadrant of each dog. A simple photographic system was developed for recording the in vivo gingival circulation depicted by fluorescein angiography just prior to surgery and then after surgery on days $1,3,7$, 10,14 , and 21 . The flaps were divided into three interproximal and two mid-buccal sites for analysis and the intracapillary and diffusion extent of dye fluorescence was accurately quantified by computerized planimetry. As healing progressed, longitudinal changes relative to presurgical baseline were analyzed by paired $t$-test. Cross-sectional comparisons utilizing Student $t$-test allowed for evaluating differences between the two suturing techniques as well as differences between interproximal versus mid-buccal sites at each postsurgical day. It was found that the simple act of raising a mucoperiosteal envelope flap initiates significant vascular trauma. Statistically significant reductions in flap circulation relative to presurgical baseline lasted for at least 3 days but persisted for 7 days at the interproximal sites. Flap diffusion (extravascular leakage) recovered sooner and extended over a significantly greater area of the flap than did intracapillary flap circulation during the early period of healing. No significant differences appeared between the two suturing techniques compared, although both may have exerted a local negative influence on the circulation until they were removed. $J$ Periodontol 1995; 66:205-210.

Key Words: Surgical flaps; sutures; vascular patency; blood circulation.

Flap procedures constitute a frequent surgical modality employed in periodontal therapy. While it is generally agreed that close flap adaptation with precise interdental union between the buccal and lingual papillae is important, the conditions necessary for healing by primary intention are difficult, if not impossible to achieve. ${ }^{1-5}$ Several factors may be responsible for this including vascular impairment within the flap or possibly an adverse effect from the sutures. The inflammatory foreign body reaction evoked in oral tissues by various suture materials has been investigated. ${ }^{6-10}$ However, since the sutures in these studies were tied or implanted in otherwise intact, non-manipulated oral

*Private practice, Farmington Hills, MI.

tDepartment of Periodontics/Prevention and Geriatrics, School of Dentistry, The University of Michigan, Ann Arbor, MI.

‡Department of Stomatology, Division of Periodontics, Dental Branch, The University of Texas-Houston, Health Science Center, Houston, TX. tissues, the influence they might impart during the actual healing of periodontal flaps can only be speculated. Furthermore, while many methods for suturing in periodontics have been recommended, ${ }^{11-15}$ the techniques have never been evaluated with regards to their vascular response or effect on the healing process. Sutures have been shown to place tissues under tension and cause circulatory stasis, both of which can retard wound healing. ${ }^{16-18}$ Therefore, it would seem that the position and method of suture placement could influence the postoperative healing of periodontal flaps.

The importance of a rich blood supply has long been recognized as an essential requirement to promote rapid and uncomplicated postoperative healing, and for this reason a number of studies have investigated revascularization following different periodontal flap procedures. ${ }^{2-5}$ Their conclusions based on histologic examination of specimens after 


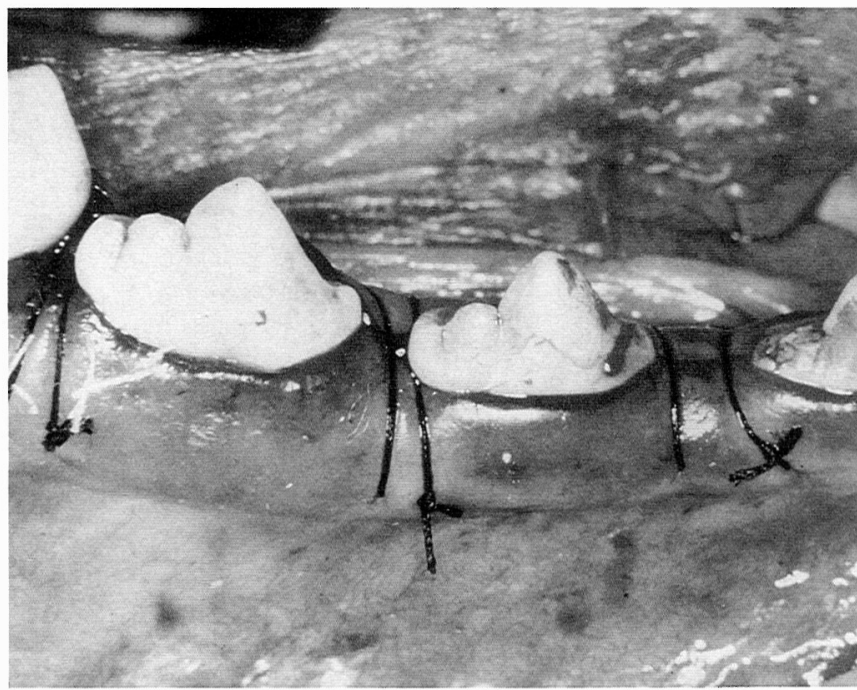

Figure 1A. Immediate postoperative flap closure using the horizontal mattress suturing technique.

vascular perfusion suggest that blood vessels remain intact and patent following surgery. Yet, another in vivo study on various incisions and modifications in periodontal flap design has shown that obvious circulatory reductions do occur. ${ }^{19}$ Though accurate quantification of the changes was not attempted, this latter study utilized fluorescein angiography which is capable of demonstrating several functional parameters of the circulation. The technique has been widely utilized for basic research and clinical application in the fields of plastic surgery and ophthalmology, though it has received little attention in periodontal research.

A simple and inexpensive photographic system for fluorescein angiography was developed for this study in order to determine the extent of vascular changes following mucoperiosteal flap surgery in the dog model, where two different suturing techniques were employed.

\section{MATERIALS AND METHODS}

This study was performed in accordance with the National Institute of Health Guide for the Care and Use of Laboratory Animals.

Four young and healthy adult mongrel dogs with slight marginal gingivitis and a full complement of teeth were selected for this study. An additional criterion for selection was that the gingival tissues appear completely pink in color, since melanin pigmentation interferes with fluorescein angiographic evaluation. At the beginning of a 2-week presurgical period the teeth were initially scaled, and plaque control was maintained throughout the study by rubber cup prophylaxes every other day. Only the mandibular posterior regions were chosen for surgery since this allowed a consistent operation to be uniformly repeated in all dogs, where reflection of both buccal and lingual gingival flaps is easy, and muscle attachments which can cause postoperative displacement are minimal. The overall intent was to eliminate, or at least minimize, any factor which could compromise healing. Any vas-

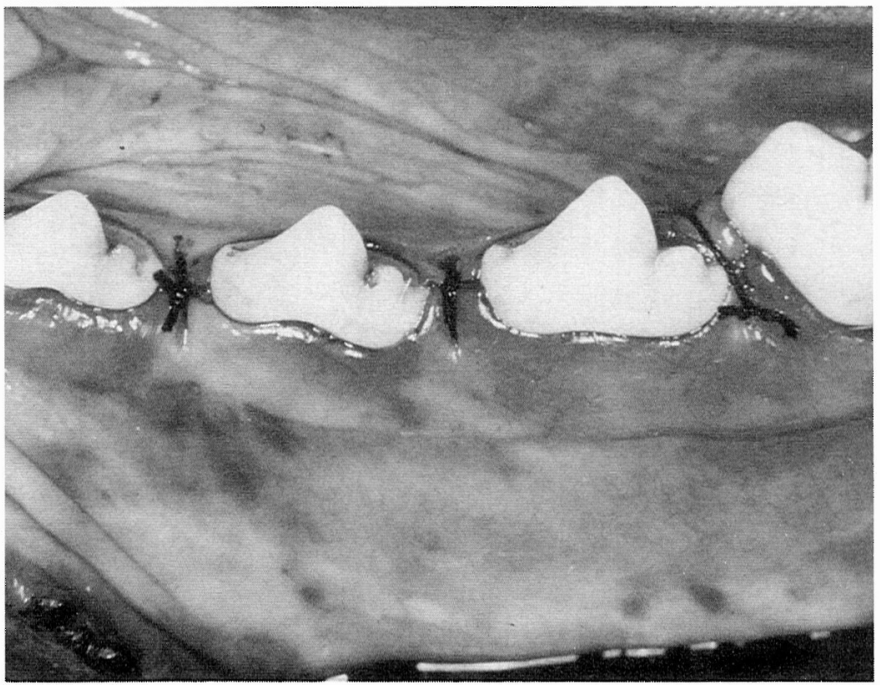

Figure 1B. Immediate postoperative flap closure using the direct interrupted suturing technique.

cular disturbance would, therefore, be directly attributable to the surgical and suturing procedures alone.

Under general anesthesia with pentobarbital sodium (Nembutal), $30 \mathrm{mg} / \mathrm{kg}$ body weight, supplemented by local anesthesia (Lidocaine $\mathrm{HCl} 2 \%$ ), buccal and lingual mucoperiosteal flaps were bluntly elevated to the mucogingival junction in both mandibular quadrants of each dog following intracrevicular incisions from the first premolar to first molar. Vertical releasing incisions were avoided and the full-thickness envelope flaps were immediately repositioned to their original location with no additional thinning or tissue plasty. Gauze pressure was maintained until all hemorrhage ceased and precise closure with 4-0 silk sutures was achieved. The direct interrupted (through-and-through) suturing technique was randomly assigned for one quadrant in each dog and the horizontal mattress (blanket) interrupted technique was used for the contralateral quadrant (Fig. 1).

\section{Fluorescein Angiography}

A simple photographic system for fluorescein angiography was developed and tested prior to this study which permitted rapid-sequence recording of the buccal gingival circulation. The system consisted of a $35 \mathrm{~mm}$ SLR camera ${ }^{\S}$ with a power winder, two mounted flash units" covered by excitation filters, " and a $90 \mathrm{~mm}$ macro lens covered by a barrier filter." The dark blue excitation filters emitted light at the low end of the visible spectrum for stimulating the circulating fluorescein, while the yellow barrier filter kept out most other wavelengths of light from being recorded on the photographic film except for the yellow-green fluorescence. The photographic system was suspended over the dogs by

§Canon Inc., Tokyo, Japan.

Vivitar Photo Electronik, GMBH, Germany.

TWratten \#47A, Eastman Kodak Company, Rochester, NY.

*Wratten \#12, Eastman Kodak Company, Rochester, NY. 


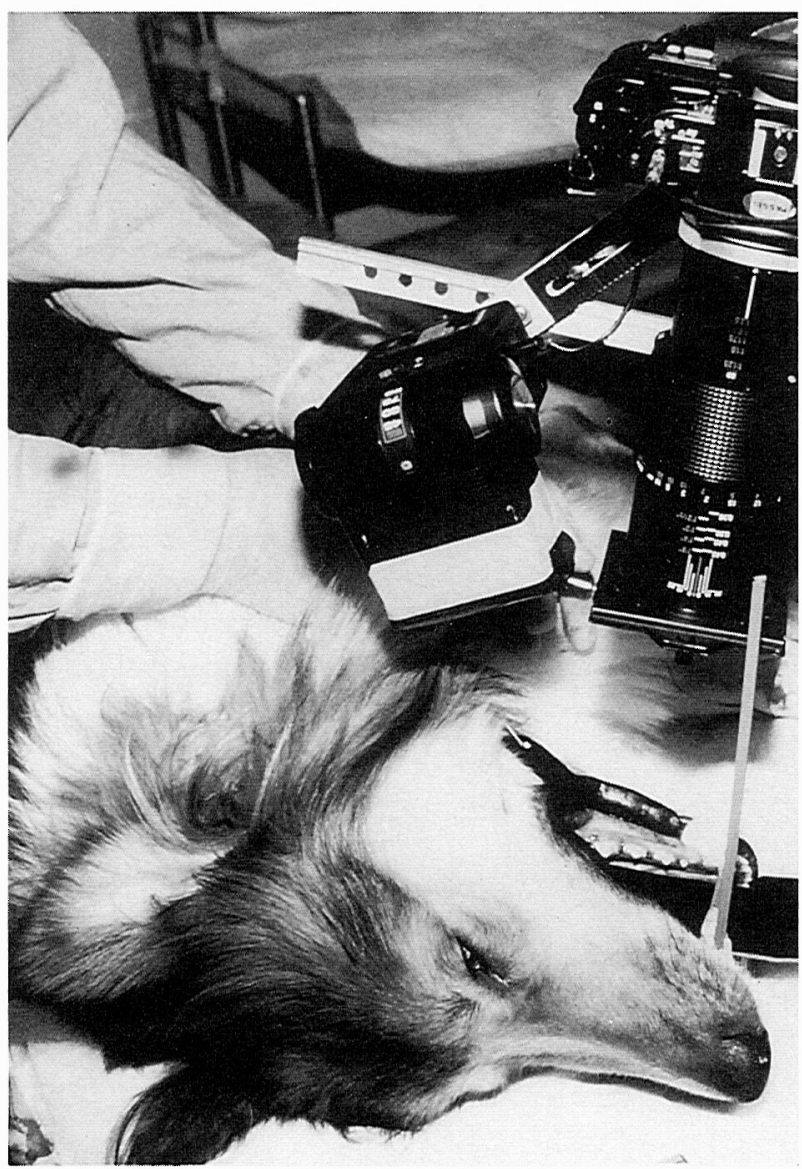

Figure 2. Photographic system developed for fluorescein angiography, suspended over dog by a tripod.

a tripod, and with the aid of fabricated occlusal stents it was focused perpendicularly onto the surgical site at a magnification of 1:1 (Fig. 2). The lens aperture was set at F4 to allow maximal light during the $1 / 60$ th second exposure and yet maintain a reasonable depth of field for clear focus at close range. Due to the excitation and barrier filters employed, there was a further reduction of available light. The use of high-speed film** was beneficial in this regard. Two flash units prevented a shadow effect, but were also specifically chosen for their ability to discharge an intense flash repeatedly at one second intervals without failing to recharge at this pace. The final result achieved by this photographic system was the obtainment of a sequential series of high quality fluorescein angiograms.

Following rapid intravenous injection of sodium fluorescein, ${ }^{\text {t十 }} 7 \mathrm{mg} / \mathrm{kg}$ body weight via a 19 gauge butterfly catheter, the first appearance, filling, and intracapillary extent of dye fluorescence was documented by serial exposures recorded initially at one second intervals. Subsequent exposures at progressively longer intervals until 3 minutes after injection recorded extravascular leakage and interstitial diffusion of the fluorescein. Baseline angiographic se-

**Ektachrome ASA 400, Eastman Kodak Company, Rochester, NY. ${ }^{\dagger+}$ Fluorescite injection 5\%, Alcon Laboratories, Inc., Fort Worth, TX.

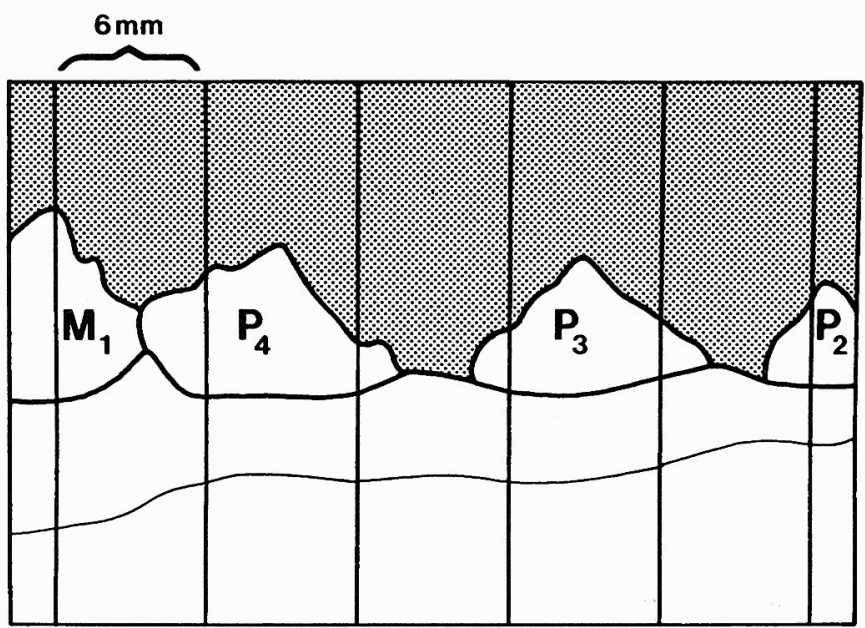

Figure 3. Method of distinguishing between the interproximal and midbuccal sites for evaluating the fluorescein angiograms.

ries were obtained immediately before surgery along with identical series at $1,3,7,10,14$, and 21 days postsurgery.

The slides representing the intracapillary and extravascular diffusion extent of dye fluorescence at all postsurgical days were selected for quantitative evaluation. This was accomplished with the aid of a computerized planimetry system incorporating a graphics tablet and digitizer screen. $¥$ The slides were projected at $20 \times$ magnification, onto a digitizer screen and each quadrant of flap surgery was divided for the purpose of evaluation into 3 interproximal and 2 mid-buccal sites of equal mesio-distal width, using an overlay, as shown in Figure 3. Both the total area within each site and the partial area showing fluorescence were traced on the screen with a cursor pen. This digitized information was transferred to a graphics tablet where a computer program integrated into the system generated square millimeter values from the irregular-shaped tracings. Finally, calculations were made to determine the percent of fluorescence within each site.

The computerized planimetry system employed was tested for reproducibility and accuracy by randomly choosing and retracing sites at a later date. The mean difference between repeat tracings of the total and fluoresced areas was .007 and $.030 \mathrm{~mm}^{2}$, respectively. Analysis of these differences by paired $t$-test showed no significance. As judged by determination of the $95 \%$ confidence interval, the error in determining percent fluorescence was only $+/-0.546 \%$.

\section{Statistical Analysis}

As an essential preliminary evaluation of the data, the intraclass correlation coefficient ${ }^{20}$ was established. It was found appropriate to consider all the sites as independent variables, as there were no greater differences between dogs than there were within any individual dog. Longitudinal changes relative to presurgical baseline as healing progressed were analyzed by paired $t$-test. Cross-sectional 
comparisons utilizing Student $t$-test allowed evaluating differences between the two suturing techniques as well as differences between interproximal versus mid-buccal sites at each postsurgical day.

\section{RESULTS}

Baseline intracapillary and diffusion fluorescence within the gingival flaps was always $100 \%$ at day 0 immediately prior to surgery. As seen in Figure 4, significant reductions in flap circulation (intracapillary fluorescence) resulted following surgery. At day 1 the mean percent intracapillary fluorescence was under $80 \%$ (Fig. 5). While improvement occurred with time, statistically significant reductions relative

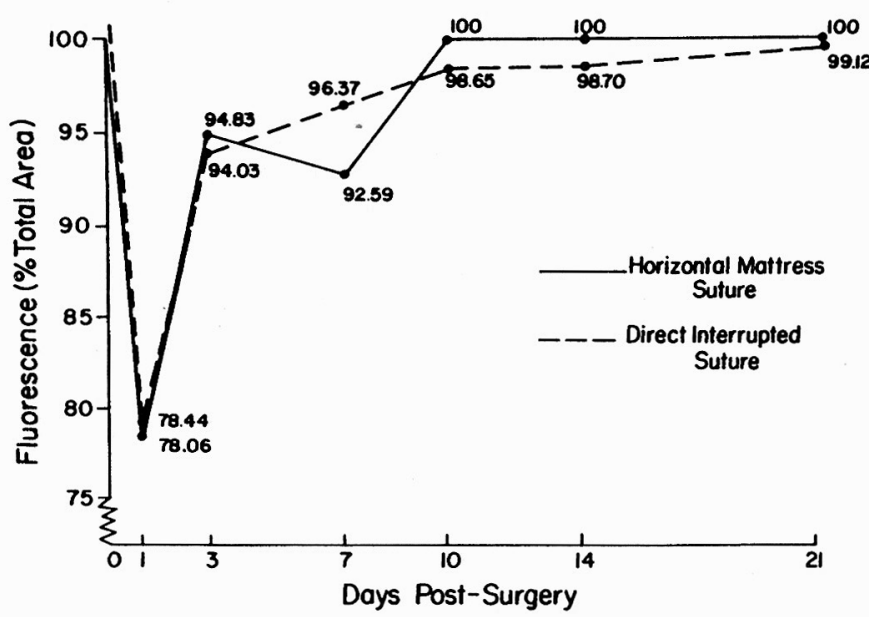

Figure 4. Mean percent intracapillary fluorescence comparing the horizontal mattress $(n=20)$ and direct interrupted $(n=20)$ sutures.

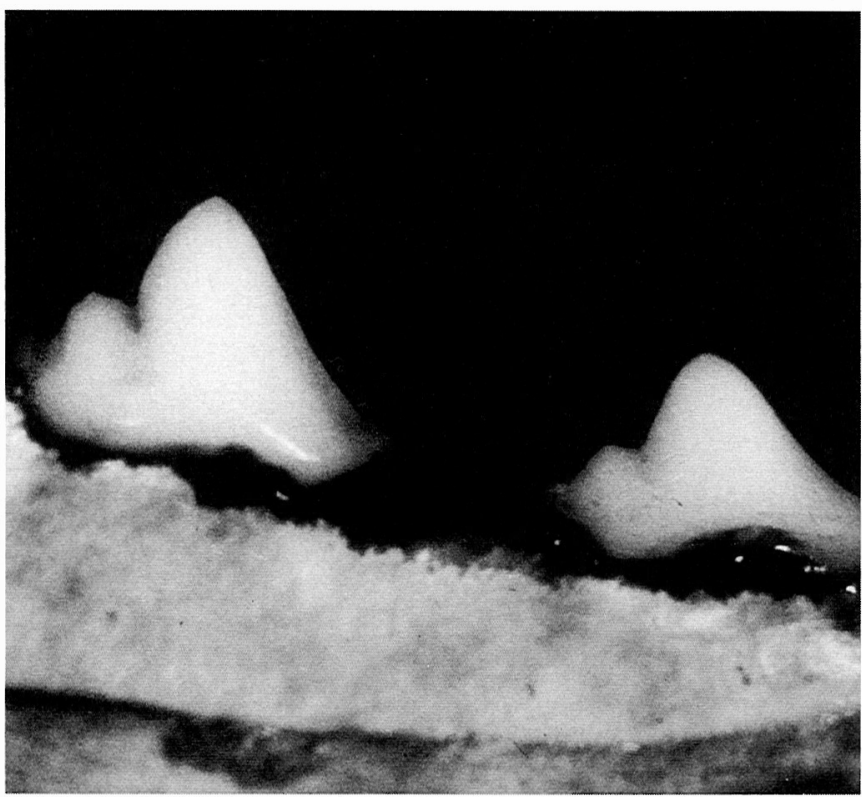

Figure 5. Typical fluorescein angiogram depicting lack of intracapillary fluorescence on post-surgical day 1 at the coronal-most portion of the gingival flap. to baseline were still evident at postsurgical days 3 and 7 , though there were no differences between the two suturing techniques at any postsurgical day.

The data were next stratified to distinguish the interproximal from mid-buccal sites, since suture-related circulatory effects may only appear in their near vicinity at the interdental papillae. As shown in Figure 6, a significant discrepancy did emerge by day 7 after surgery. Up until this time at postsurgical days 1 and 3 , both sites had significant circulatory reductions relative to baseline. By day 7 , however, the mid-buccal sites had regained complete intracapillary fluorescence, while the interproximal sites continued to demonstrate reductions which were significant relative to baseline.

The interproximal sites alone were subsequently analyzed to reveal possible differences between the horizontal mattress and direct interrupted suturing techniques (Fig. 7).

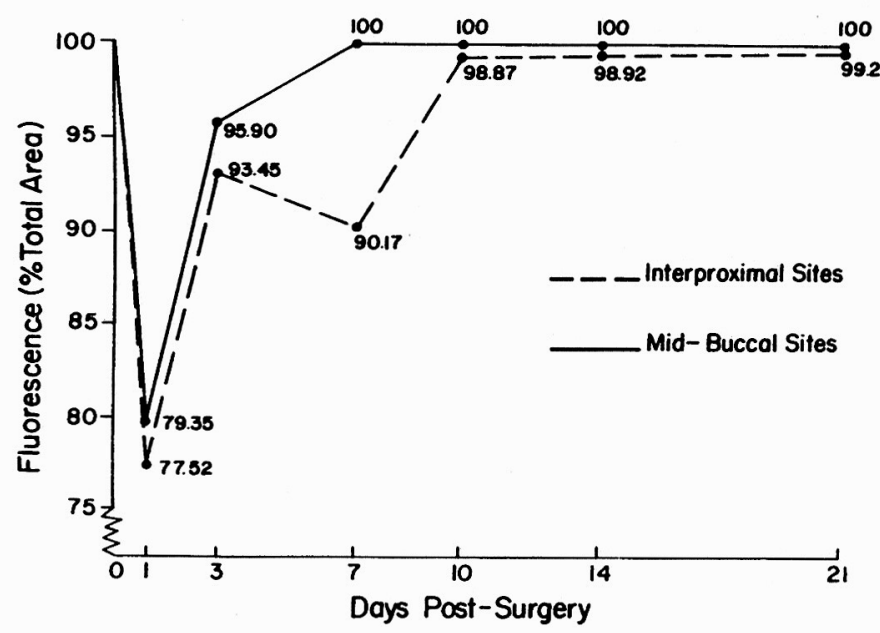

Figure 6. Mean percent intracapillary fluorescence comparing the interproximal $(n=24)$ and mid-buccal $(n=16)$ sites.

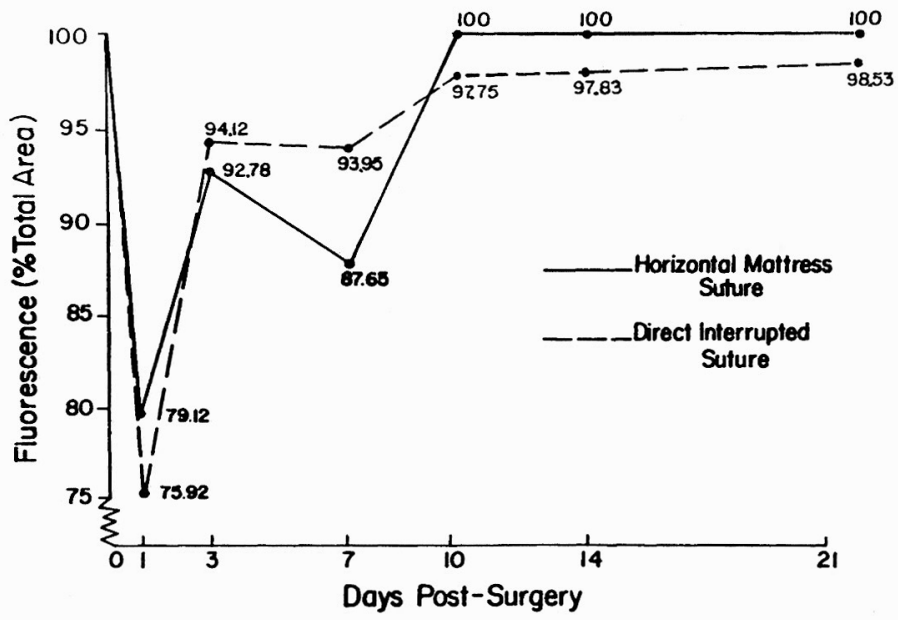

Figure 7. Mean percent intracapillary fuorescence comparing the horizontal mattress $(n=12)$ and direct interrupted $(n=12)$ sutures at the interproximal sites. 
But again, no statistically significant differences between the sutures were found at any given day.

There was evidence, however, implicating the sutures as being at least partly responsible for the circulatory disturbances observed. As can be seen in Figure 6, there is actually a regression in intracapillary fluorescence between post-surgery days 3 and 7 , but only at the interproximal sites. Figure 7 further shows that both suture types were associated with this circulatory phenomenon. Some sutures were lost prematurely in this study between the fourth and seventh postsurgical day (roughly equal numbers of both types in all dogs). Table 1 shows that the extent of intracapillary fluorescence at the interproximal sites 7 days postsurgery was greater when sutures were missing than when they were still present. Analysis of this difference by Student $t$-test, while not quite significant at the $5 \%$ level, is suggestive of a trend that sutures may induce a subtle pathologic reaction, and when left in place for one week may retard the circulation around the suture material near the flap margin.

The relationship between intracapillary flap circulation and extravascular flap diffusion or leakage is depicted graphically in Figure 8. Significantly greater areas of fluorescence were achieved at days 1 and 3 by extra-vascular diffusion over that achieved by intracapillary circulation.

Table 1. Mean Percent Intracapillary Fluorescence on Day 7 Postsurgery at the Interproximal Sites

\begin{tabular}{lcc}
\hline & Sutures & Sutures \\
& Present & Absent \\
\hline Mean & 84.16 & 97.04 \\
Standard deviation & 17.28 & 3.82 \\
$\mathrm{n}$ & 8 & 7 \\
& Mean difference & -12.88 \\
& Student $t$-test & -1.922 \\
& $P$ (test values) & .08 \\
\hline
\end{tabular}

$P=$ Test Value

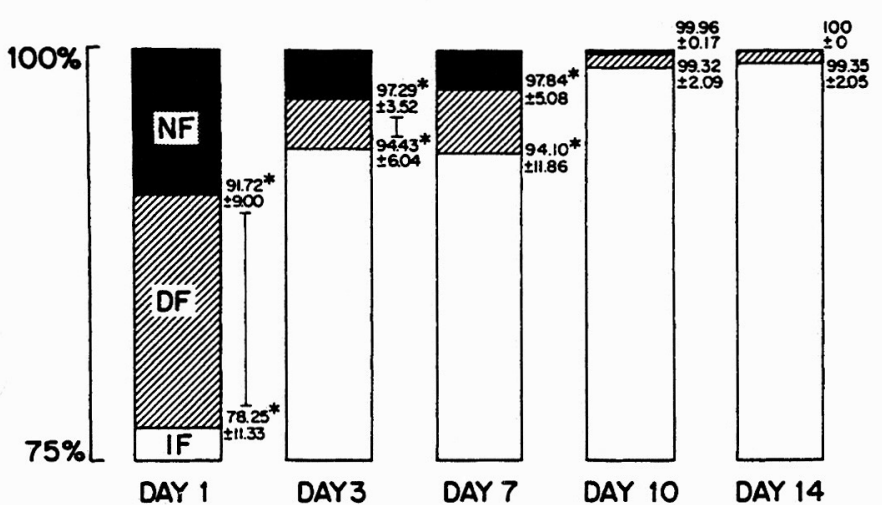

$$
\begin{aligned}
& \text { * Signif diff. from baseline } \\
& \left(10 \% \% \text { Fluorescence), paired t-test } \quad \begin{array}{l}
\text { Signif diff. between intracapillary } \\
\text { and diffusion flucrescence, } \\
\text { student's t-test }(p<05)
\end{array}\right.
\end{aligned}
$$

Figure 8. Mean percent fluorescence at the intracapillary (IF) and diffusion (DF) phase ( $n=40 ; N F=$ nonfluoresced flap portion).
At day 1 postsurgery, over $90 \%$ of the total flap area already demonstrated diffusion fluorescence. Even so, for the first 7 days after surgery statistically significant portions of the flaps remained nonfluoresced relative to presurgical baseline, despite the extra flap area reached through diffusion.

\section{DISCUSSION}

This fluorescein angiography study shows that significant reductions in flap circulation result following periodontal flap surgery. Using the same technique, Mörmann and Ciancio ${ }^{17}$ also noted an impaired circulation in periodontal flaps, although their main concern focused on the importance of maintaining a good blood supply from vessels entering a pedicle flap at its base. They restated an old principle from plastic surgery that a flap's length to width ratio should not exceed 2:1. While it seems logical and appears to make biological sense that increasing the flap's width at its base will increase the blood supply and support a greater flap length, this classic 2:1 concept was proven a fallacy by Milton ${ }^{21}$ as being too simplistic. Furthermore, it cannot be assumed that major nutrient vessels enter the base of gingival flaps at regular intervals. Jeffcoat et al. ${ }^{22}$ showed the mandibular gingival vasculature in beagle dogs with arterial vessels traversing somewhat obliquely in a general posterior to anterior direction. Vertical releasing incisions were intentionally avoided in the present study and yet it was found that the simple act of raising a full thickness envelope flap created a significant circulatory embarrassment for up to 7 days postsurgery. While it is certainly prudent to avoid long and narrow pedicle flaps in periodontal surgery, alternate vascular sources deserve recognition.

In this study, it was found that, during the early days of healing after surgery, significantly greater portions of the flaps gained fluorescence by extravascular diffusion over that achieved by the intracapillary circulation. This may have provided an important source of nutrition for the coronal portions of the flaps prior to the time when an active circulation was restored throughout. It would therefore be appropriate to recognize that periodontal flaps may survive during the early postsurgical period in a manner similar to that of gingival grafts. Such a concept gains support from the study by Dibbell et al. ${ }^{23}$ who placed cellophane barriers beneath pedicle skin flaps to demonstrate that they acted much more like grafts at their distal portions farthest away from the base. The fluorescein angiograms in the present study revealed that intracapillary circulation was often absent near the location of the sutures. These reductions occurred for both suturing techniques at the interproximal sites for as long as the sutures remained in place. While sutures have been shown to have no effect on healing when a good blood supply exists and tension is minimal, ${ }^{24}$ they could act as a complicating factor when placed in areas with a marginal blood supply threatened by ischemia. A suturerelated effect or perhaps just the reduction in circulation which occurs at the flap margins and papillae farthest from 
the base may explain the common clinical observation after periodontal flap surgery of interproximal soft tissue deformities which require prolonged periods of time to repair and remodel, despite attempts at precise closure to encourage healing by primary intention.

\section{Conclusions}

Within the limits of this study it can be concluded that:

1. The simple act of raising a mucoperiosteal envelope flap initiates a significant vascular embarrassment.

2. A lack of circulation at the most coronal portion of the flaps lasts for at least 3 days but persists for 7 days at the interproximal regions.

3. Flap diffusion recovers sooner than flap circulation, and during the early period of healing diffusion extends significantly farther towards the coronal margin.

4. No apparent differences between the horizontal mattress and direct interrupted suturing techniques exist regarding different effects on flap circulation, though both may exert a localized negative influence during the early period of healing.

\section{REFERENCES}

1. Caffesse RG, Ramfjord SP, Nasjleti CE. Reverse bevel periodontal flaps in monkeys. J Periodontol 1968; 39:219-235.

2. Caffesse RG, Castelli WA, Nasjleti CE. Vascular response to modified Widman flap surgery in monkeys. $J$ Periodontol 1981; 52:1-7.

3. Kon S, Caffesse RG, Castelli WA, Nasjleti CE. Revascularization following a combined gingival flap-split thickness flap procedure in monkeys. J Periodontol 1984; 55:345-351.

4. Caffesse RG, Kon S, Castelli WA, Nasjleti CE. Revascularization following the lateral sliding flap procedure. J Periodontol 1984; 55: $352-358$.

5. Kon S, Novaes AB, Ruben MP, Goldman HM. Visualization of the microvascularization of the healing periodontal wound. IV. Mucogingival surgery: Full thickness flap. J Periodontol 1969; 40:441-456.

6. Bergenholtz A, Isaksson $\mathrm{B}$. Tissue reactions in the oral mucosa to catgut, silk, and Mersilene sutures. Odont Revy 1967; 18:237-250.

7. Castelli WA, Nasjleti CE, Caffesse RG, Diaz-Perez R. Gingival response to silk, cotton and nylon suture materials. Oral Surg Oral Med Oral Pathol 1978; 45:179-185.

8. Castelli WA, Nasjleti CE, Diaz-Perez R, Caffesse RG. Cheek mucosa response to silk, cotton and nylon suture materials. Oral Surg Oral Med Oral Pathol 1978; 45:186-189.

9. Lilly GE. Reaction of oral tissues to suture materials. Oral Surg Oral Med.Oral Pathol 1968; 26:128-133.

10. Lilly GE, Armstrong JH, Salem JE, Cutcher JL. Reaction of oral tissues to suture materials. Part II. Oral Surg Oral Med Oral Pathol 1968; 26:592-599.

11. Becker W, Becker BE, Prichard JF, et al. Root isolation for new attachment procedures. A surgical and suturing method: Three cases reports. J Periodontol 1987; 58:819-826.

12. Dahlberg WH. The Dental Clinics of North America, Vol 13. Philadelphia: WB Saunders Co.; 1969; 149-158.

13. Grupe $\mathrm{H}$, Warren $\mathrm{R}$. Repair of gingival defects by a sliding flap operation. J Periodontol 1956; 27:92-95.

14. Heijl L, Wennstrom J, Lindhe J. Periodontal surgery: Techniques for periodontal pockets; suturing techniques. In: Lindhe J, ed, Textbook of Clinical Periodontology. Copenhagen: Munksgaard; 1983; 386388.

15. Carranza FA, Takei HH. The periodontal flap; suturing techniques. In: Carranza FA ed, Glickman's Clinical Periodontology. Philadelphia: W.B. Saunders Company; 1979; 827-837.

16. Edlich RF, Panek PH, Rodheaver GT, et al. Physical and chemical configuration of sutures in the development of surgical infections. Ann Surg 1973; 177:679-683.

17. Mörmann W, Ciancio SG. Blood supply of human gingiva following periodontal surgery. A fluorescein angiographic study. $J$ Periodontol 1977; 48:681-692.

18. Postlethwait RW, Willigan DA, Ulin AW. Human tissue reaction to sutures. Ann Surg 1975; 181:144-151.

19. Mörmann W, Bernimoulin J-P, Schmid MD. Fluorescein angiography of free gingival autografts. J Clin Periodontol 1975:2:177-189.

20. Cohen ME, Cecil JC. Intraclass correlation and the application of analysis of variance to dental data. $J$ Dent Res 1983; 62:322-327.

21. Milton SH. Pedicled skin flaps: The fallacy of the length:width ratio. Br J Surg 1970; 57:502-506.

22. Jeffcoat MK, Kaplan ML, Rumbaugh CL, Goldhaber P. Magnification angiography in beagles with periodontal disease. $J$ Periodont Res 1982; 17:294-299.

23. Dibbell DG, Hedburg JR, McGraw JB, et al. A quantitative examination of the use of fluorescein in predicting viability of skin flaps. Ann Plast Surg 1979; 3:101-106.

24. Myers MB. Prediction of skin sloughs at the time of operation with the use of fluorescein dye. Surgery 1962; 51:158-165.

Send reprint requests to: Dr. Raul G. Caffesse, Dental Branch, The University of Texas-Houston HSC, 6516 John Freeman Avenue, Room 309, Houston, TX 77030.

Accepted for publication August 22, 1994. 